TITLE:

\title{
Atomistic simulation of the effects of hydrogen on the mobility of edge dislocation in alpha iron
}

\section{AUTHOR(S):}

Taketomi, Shinya; Matsumoto, Ryosuke; Miyazaki, Noriyuki

\section{CITATION:}

Taketomi, Shinya ... [et al]. Atomistic simulation of the effects of hydrogen on the mobility of edge dislocation in alpha iron. Journal of Materials Science 2008, 43(3): 1166-1169

\section{ISSUE DATE:}

2008-02

URL:

http://hdl.handle.net/2433/88962

\section{RIGHT:}

The original publication is available at www.springerlink.com; この論文 は出版社版でありません。引用の際には出版社版をご確認ご利用くだ さい。; This is not the published version. Please cite only the published version. 


\section{Atomistic Simulation of the Effects of Hydrogen on the Mobility of Edge Dislocation in Alpha Iron

\author{
Shinya Taketomi ${ }^{1,2}$, Ryosuke Matsumoto ${ }^{1,2}$ and Noriyuki Miyazaki ${ }^{1,2}$ \\ ${ }^{I}$ Department of Mechanical Engineering and Science, Graduate School of Engineering, Kyoto University, \\ Yoshida-Honmachi, Sakyo-ku, Kyoto, 606-8501, Japan \\ ${ }^{2}$ Visiting Researcher, National Institute of Advanced Industrial Science and Technology (AIST)
}

Despite extensive investigations concerning hydrogen embrittlement mechanisms, not all the effects of hydrogen on material properties have been clarified. One of the effects of hydrogen is an enhancement of plasticity localisation and is known as the hydrogen enhanced localised plasticity (HELP) mechanism [1]. An in situ observation of the dislocations under a hydrogen gaseous environment was performed using a transmission electron microscope (TEM), which revealed the reduction of the distance between dislocations when hydrogen gas was added into the environmental cell $[2,3]$. Such a plasticity localisation is observed in a large number of materials and slip systems [4]. Therefore, these experimental results are considered to be powerful evidence of HELP. Although the experimental observations show only the reduction of the distance between dislocations under a hydrogen gaseous environment, the precise reason for this reaction is still unclear. Elasticity analyses suggest that hydrogen shields the elastic stress around edge dislocations and results in dislocation localisation [5]. However, hydrogen concentration was extremely high in that particular analysis. Furthermore, the analyses could not take into account the hydrogen accumulation at dislocation core, in contrast with the results of the ab initio study, which show that the dislocation core has strong binding to hydrogen [6]. Moreover, the $a b$ initio study showed that hydrogen lowers the Peierls stress for pure aluminium. In this study, analyses are conducted based upon atomistic models composed of alpha iron in order to investigate the effect of hydrogen on the mobility of edge dislocations. First, the hydrogen distribution around an edge dislocation is accurately estimated based on the embedded atom method (EAM) potential fitted by Wen et. al [7]. Second, the energy barriers for edge dislocation motion are investigated using the nudged elastic band (NEB) method [8,9]. Finally, the stress fields around the edge dislocation in the presence of hydrogen are calculated using the molecular statics (MS) method.

The geometrical shapes and crystallographic orientations of the analysis model are shown in Fig. 1. Slip occurs on the (112) plane, and the slip direction is along the [111] axis. This slip system is reported to be sensitive to hydrogen embrittlement [10]; moreover, according to the theory of elasticity, only an edge component that yields hydrostatic stress is expected to interact with hydrogen atoms. In order to introduce an edge dislocation into the model, the atomic plane shown in Fig. 1 is removed, and the structure is then relaxed by the conjugate gradient (CG) method. The system contains 8,054 Fe atoms, and the unit cell size is $11.05 \mathrm{~nm}, 4.91 \mathrm{~nm}$ and $2.02 \mathrm{~nm}$ in the $\mathrm{x}, \mathrm{y}$ and $\mathrm{z}$ directions, respectively. Periodic boundary conditions are applied in the $\mathrm{x}$ and $\mathrm{z}$ directions. In this system, dislocation density is approximately $0.018 \mathrm{~nm}^{-2}$. The EAM potential for the $\mathrm{Fe}-\mathrm{H}$ system proposed by Wen et al. [9], which is reported to be the most reliable interatomic potential compared to previously proposed interatomic potentials [11], is adopted in this study. The hydrogen occupation site in a perfect bcc lattice may be characterised into two sites: a tetrahedral site (T-site) and an octahedral site (O-site). The T-site is reported to be the most stable site for a hydrogen atom in alpha iron [12]. However, the crystallographic structure around an edge dislocation does not maintain a perfect bcc structure; therefore, it is difficult to locate the actual occupation site of hydrogen. In this study, T-sites for a perfect bcc lattice correspond to the vertices of the Voronoi polyhedron of the bcc structure. Thus, the positions of hydrogen occupation sites in the system are estimated according to the vertices of the Voronoi polyhedron. The energy barrier for dislocation motion is estimated using the NEB method. This method is one of the transition state theories and enables us to obtain the minimum energy path (MEP) and the minimum energy barrier for the reaction. The NEB method requires the atomistic configurations at the initial and final states. Both state models are generated by the abovementioned method, and the dislocation at the final state is positioned $1 b$ forward along the $\mathrm{x}$ axis slip direction (Fig. 1) compared to the initial state. Here, $b$ is Burger's vector.

A hydrogen atom is introduced into each occupation site using Voronoi tessellation, and the structure is relaxed using the CG method. The distribution of the potential-energy increment at each hydrogen occupation site is shown in Fig. 2 (a). Here, the energy increment is defined by the energy difference due to the energy of hydrogen occupation within the T-site of the perfect lattice. Owing to the structural asymmetry of the (112)[111] dislocation in alpha iron, the potential-energy increment shows an asymmetrical distribution. Furthermore, typical regions with decreased potential-energy increments are the dislocation core and the slip plane. Meanwhile, the hydrogen trap activation energy at the dislocation was experimentally examined [13] $\left(4.45 \times 10^{-20} \mathrm{~J}\right)$, and the resultant value was in good quantitative agreement with our analytical results. The probability of hydrogen existing at each occupation site is expressed by the following equation. 


$$
P_{j}=\frac{\exp \left(-\Delta E_{j} / k T\right)}{\sum_{i=1}^{n} \exp \left(-\Delta E_{i} / k T\right)},
$$

where $P_{j}$ is the probability of hydrogen occupation at the $j^{\text {th }}$ site, $\Delta E_{j}$ is the potential-energy increment at the $j^{\text {th }}$ site, $k$ is Boltzmann's constant, $T$ is absolute temperature and $n$ is the total number of occupation sites in the system. Although $n$ should be the number of whole hydrogen occupation sites including O-sites, the probability of O-site occupation is assumed to be negligible in this analysis. The probability of hydrogen existence at $300 \mathrm{~K}$ calculated from equation (1) is shown in Fig. 2 (b). According to this result, a stable hydrogen atom exists near the dislocation core and on the slip plane, in contrast to the Cottrell atmosphere based on the theory of elasticity.

The energy barrier for an edge dislocation motion of $1 b$-length under the thermal activation process without hydrogen is investigated using the NEB method. The sequential characteristic of the total potential-energy of the system during dislocation motion is shown in Fig. 3 (a). This reaction shows a single energy peak, and the energy barrier is $2.648 \times 10^{-20} \mathrm{~J}$. A hydrogen atom is then introduced into the dislocation core, which is the most stable site for hydrogen. Two types of analyses are conducted: (b) with a hydrogen atom at the dislocation core in the initial state and the dislocation moving forward by $1 b$ and (c) with a hydrogen atom $1 b$ ahead of the initial dislocation and the dislocation moving to the hydrogen atom. The case in which a hydrogen atom at a dislocation core migrates along with the dislocation is not considered, because the transition path of hydrogen diffusion that occurs alongside dislocation is very difficult to distinguish. Thus, the two abovementioned basic cases are calculated to focus on the fundamental effects of hydrogen on dislocation mobility. The structure of the initial and final states that include a hydrogen atom is relaxed by the CG method to stabilise the system. The hydrogen concentration of this system is 2.24 mass ppm, and the number of hydrogen atoms per unit length of dislocation line is $0.49 \mathrm{~nm}^{-1}$. The analytical results are shown in Fig. 3. The energy barriers obtained by the NEB method are (b) $2.35 \times 10^{-20} \mathrm{~J}$ and (c) $1.18 \times 10^{-20} \mathrm{~J}$. These values are lower than that for the previously described system without hydrogen $\left(2.648 \times 10^{-20} \mathrm{~J}\right)$. It is therefore clear that the hydrogen atom contributes to decreasing the energy barrier for dislocation motion in this analytical condition. Thus, the mobility of the edge dislocation increases.

The mechanism in which hydrogen atoms around a dislocation shield the stress field of the dislocation is known as the hydrogen shielding effect [5]. The shear stress field around an edge dislocation is investigated based on the previously described atomistic model. The stress field is calculated using the MS method, and the convergence calculation is performed using the CG method. The shear stress distribution around the dislocation on the slip plane is shown in Fig. 4. Even if the number of hydrogen atoms per unit length of dislocation line is increased up to $7.35 \mathrm{~nm}^{-1}$, a significant difference in stress distribution is not observed.

In summary, this study shows that a stable hydrogen site existing around a (112)[111] edge dislocation in alpha iron is limited to the dislocation core and the exact slip plane around it. This result indicates that the hydrogen distribution around the dislocation core does not agree with the theory of elasticity. Under low hydrogen concentration conditions $\left(0.49 \mathrm{~nm}^{-1}\right.$ hydrogen atoms per unit length of dislocation line $)$, a decrease in the energy barrier for dislocation motion was detected, which could be one reason for the observed HELP mechanism. On the other hand, the interaction force between dislocations does not show significant difference under these analysis conditions.

Acknowledgements This research was performed as part of the Fundamental Research Project on Advanced Hydrogen Science funded by the New Energy and Industrial Technology Development Organization (NEDO). The research has also been partially supported by the ENEOS Hydrogen Trust Fund and the Ministry of Education, Science, Sports and Culture's Grant-in-Aid for Young Scientists (A), 19686013, 2007.

[1] C. D. Beachem, Metall. Trans. 3, 2 (1972) 437.

[2] P. J. Ferreira, I. M. Robertson, H. K. Birnbaum, Acta mater. 46, 5 (1998) 1749.

[3] P. J. Ferreira, I. M. Robertson, H. K. Birnbaum, Acta mater. 47, 10 (1999) 2991.

[4] H. K. Birnbaum, I. M. Robertson, P. Sofronis, D. Teter, in "Corrosion-Deformation Interactions" edited by T. Magnin, Inst. of Mat., (1997) 172.

[5] P. Sofronis, H. K. Birnbaum, J. Mech. Phys. Solids 43, 1 (1995) 49. 
[6] G. Lu, Q. Zhang, N. Kioussis, E. Kaxiras, Phys. Rev. Lett. 87, 9 (2001) 095501.

[7] M. Wen, X. J. Xu, S. Fukuyama, K. Yokogawa, J. Mater. Res., 16, 12 (2001) 3496.

[8] G. Henkelman, H. Jónsson, J. Chem. Phys. 113, 22 (2000) 9978.

[9] H. Jónsson, G. Mills, K. W. Jacobsen, in "Classical and Quantum Dynamics in Condensed Phase Simulations", edited by B. J. Berne et al., World Scientific, Singapore, (1998) 385.

[10] P. Bastien, P. Azou, Compt. Rend. Acad. Sci., 232 (1951) 1845.

[11] N. Miyazaki, R. Matsumoto, K. Nishimura, S. Matsumoto, Proc. International Hydrogen Energy Development Forum, (2007) 125.

[12] Y. Fukai, J. Less-Common Metals, 101 (1984) 1.

[13] W. Y. Choo, J. Y. Lee, Metall. Trans. A, 13A (1982) 135.

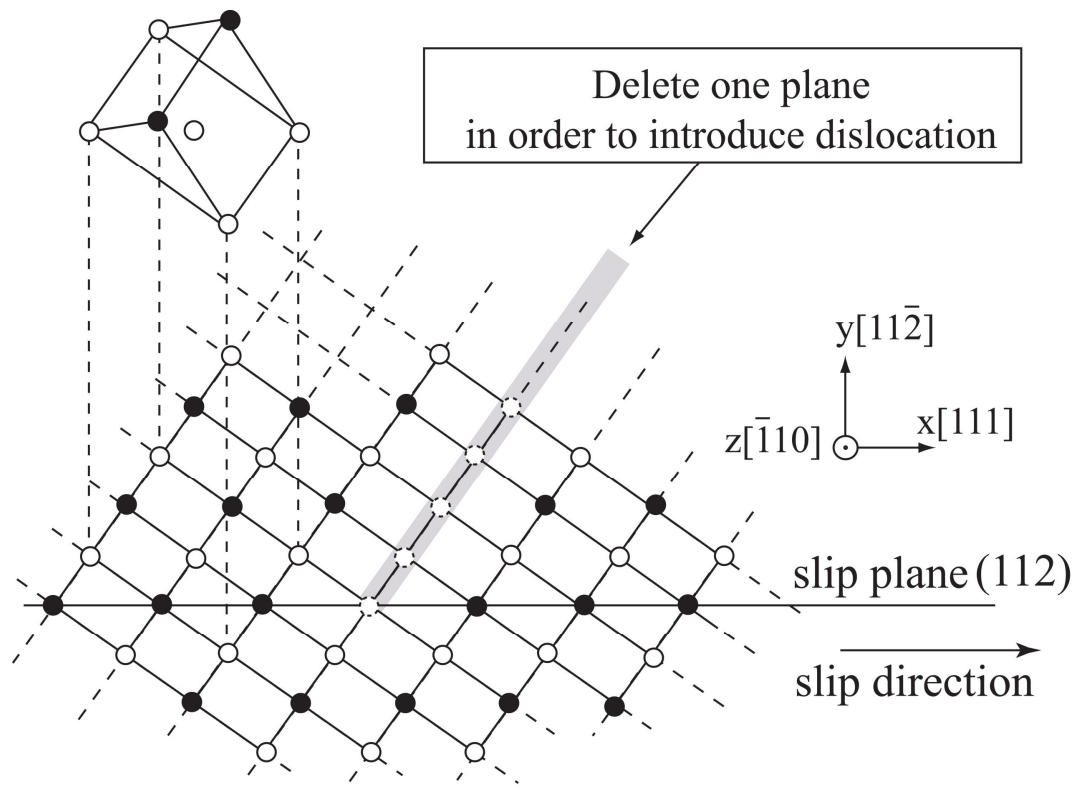

Fig.1 Atomistic simulation model within an edge dislocation. 

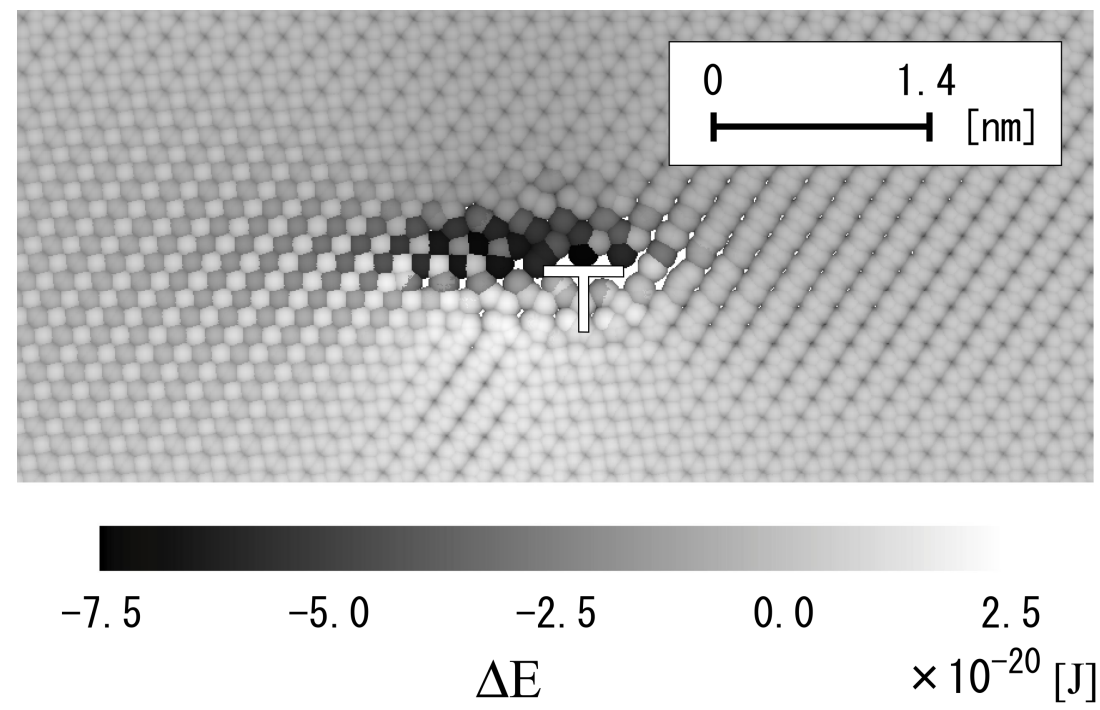

(a)

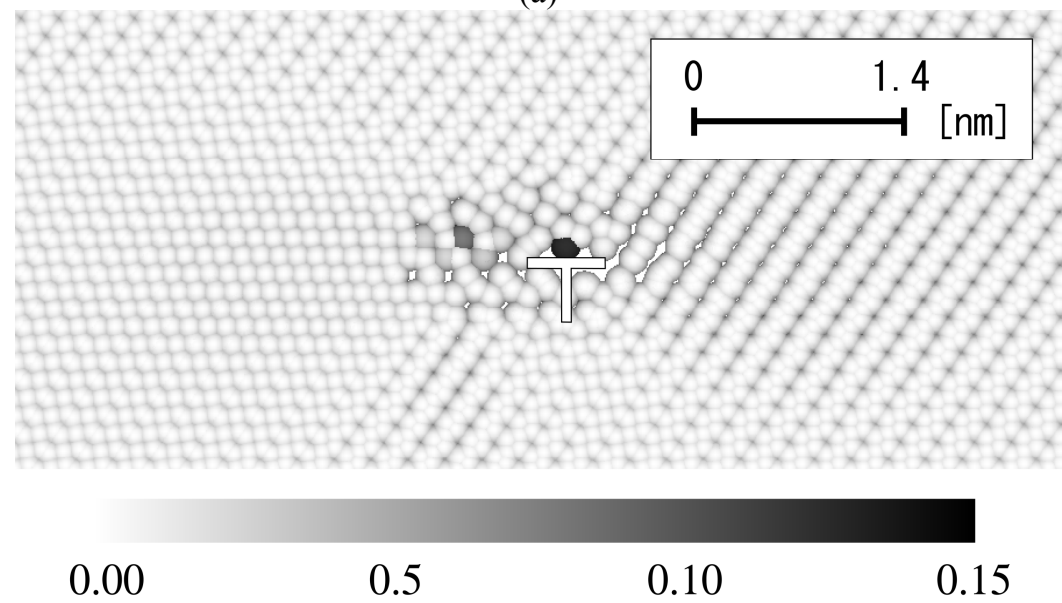

(b)

Fig. 2 (a) Distribution of potential-energy increment for each occupation site of hydrogen. (b) Probability of hydrogen occupation. 


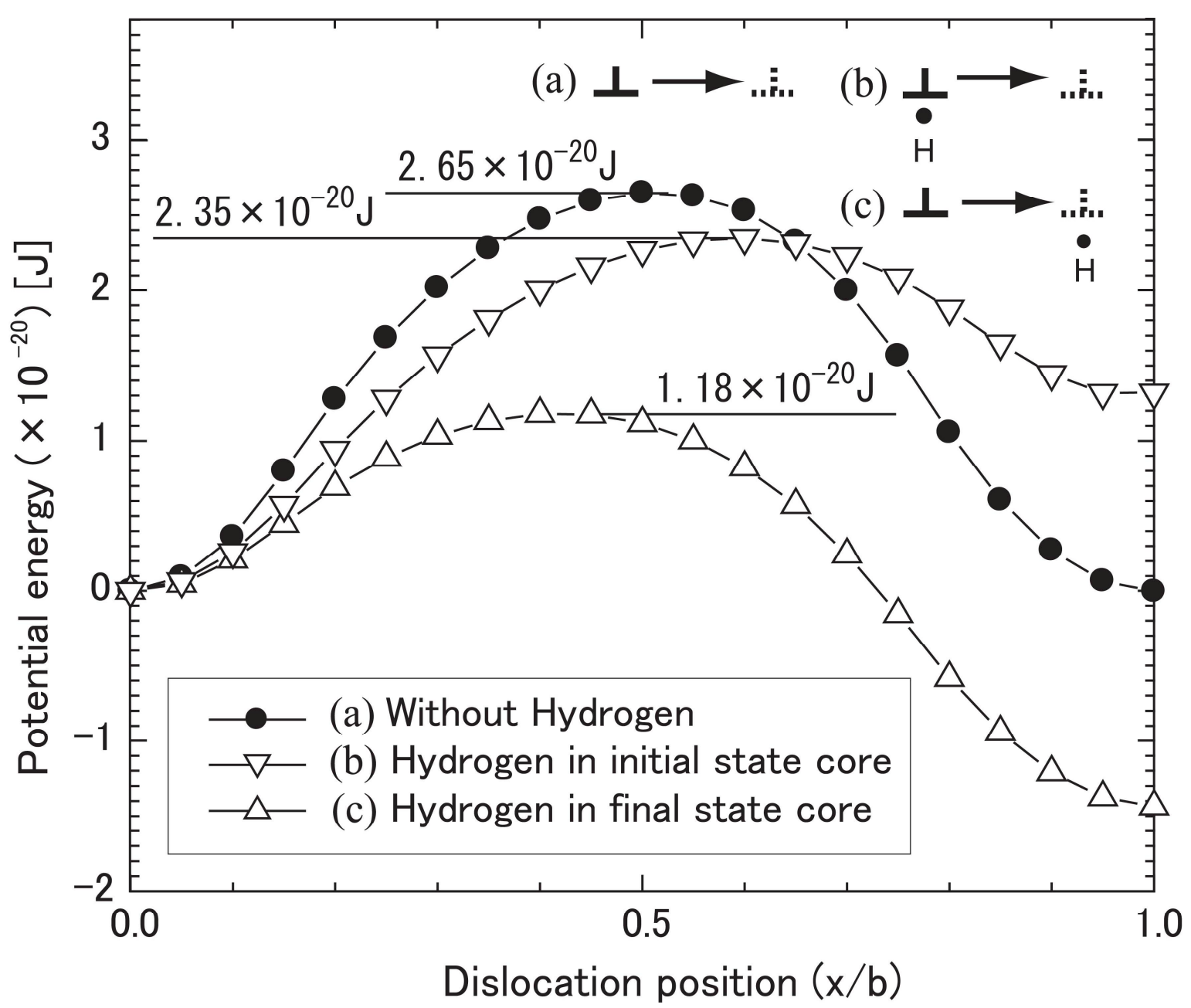

Fig. 3 Variation of energy barrier with dislocation motion: (a) without hydrogen $\left(2.65 \times 10^{-20} \mathrm{~J}\right)$, (b) with hydrogen atom at dislocation core in initial state and dislocation moving $1 b$ forward $\left(2.35 \times 10^{-20} \mathrm{~J}\right)$, and (c) with hydrogen atom $1 b$ distance ahead of initial dislocation and dislocation moving to hydrogen atom $(1.18 \times$ $\left.10^{-20} \mathrm{~J}\right)$. 


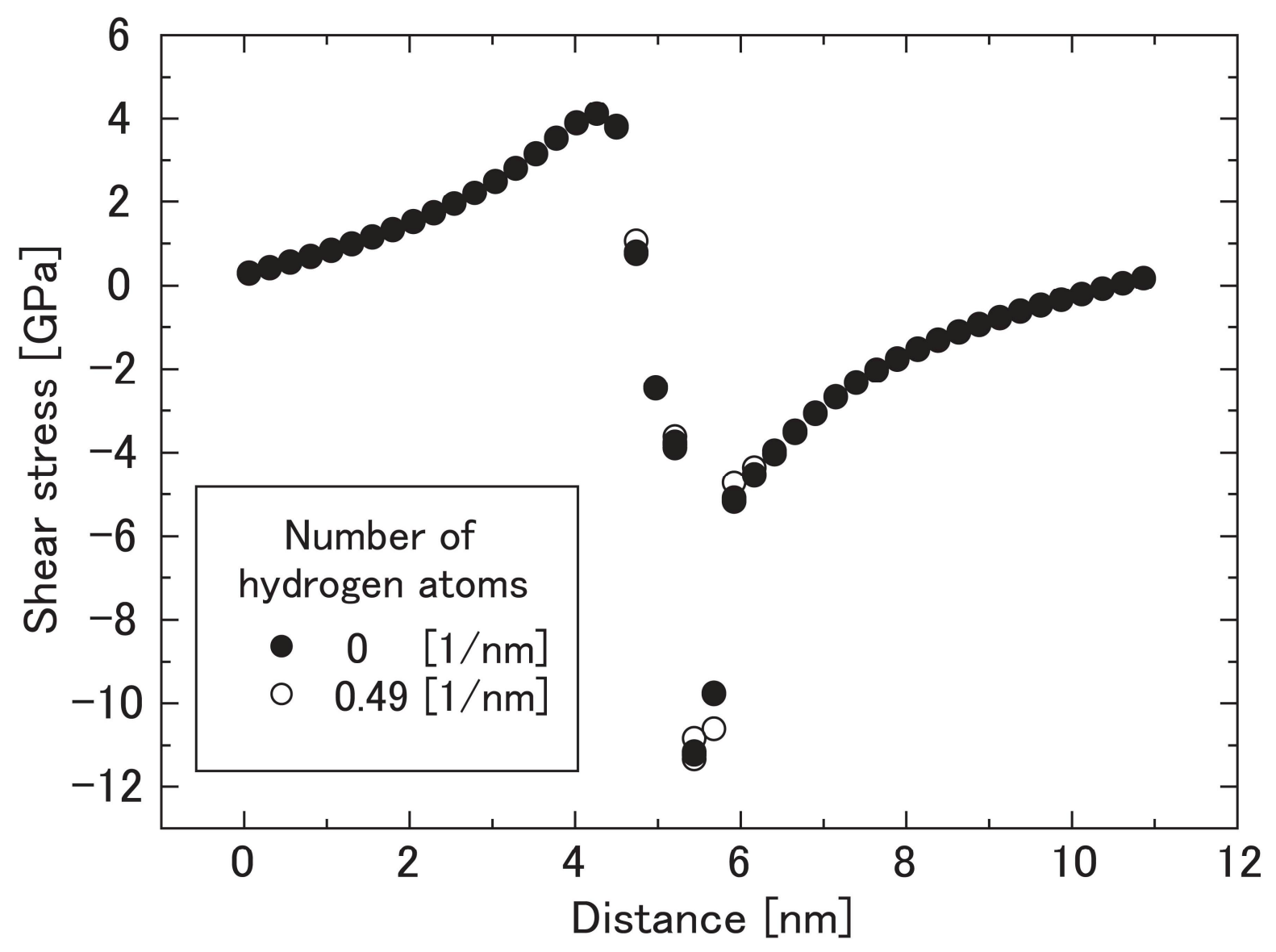

Fig. 4 Shear stress distribution along slip plane near dislocation core. 